\title{
Study of plasma flow reversal in the near-Earth plasma sheet using numerical computations
}

\author{
Koji Kondoh* and Tohru Shimizu
}

\begin{abstract}
Tailward flow in the near-Earth plasma sheet associated with a rebound of the earthward bursty bulk flow (BBF) is investigated using three-dimensional magnetohydrodynamics simulations of magnetic reconnection in the magnetotail on the basis of the spontaneous fast reconnection model. In order to investigate the properties of this tailward flow, virtual satellites are located at different positions in the plasma sheet within the simulation region, so that we can directly observe the temporal variations of plasma quantities in accordance with the growth and preceding the flow reversal associated with the magnetic reconnection. The time profile of the plasma flow velocity in the course of the BBF depends on the satellite position. Furthermore, the time profile of the magnetic field strength in the course of the reverse flow depends on the satellite position in the dawn-dusk direction. As a result of the rebound of the earthward flow, the accumulation of the plasma density and the plasma pressure is observed at any position in the plasma sheet during the interval between the BBF and the reverse flow.
\end{abstract}

Keywords: Magnetic reconnection; Bursty bulk flow; Flow reversal; MHD simulation

\section{Correspondence/findings Introduction}

Magnetic reconnection plays an essential role in large dissipative events observed in space plasmas. We have proposed the spontaneous fast reconnection model and demonstrated by magnetohydrodynamic (MHD) simulations that the fast reconnection mechanism can be realized as an eventual solution by a nonlinear instability due to the positive feedback between current-driven anomalous resistivities and reconnection flows (Kondoh et al. 2011; Ugai and Kondoh 2006). The three-dimensional (3D) spontaneous fast reconnection model has been successfully applied to geomagnetic substorms. For instance, the traveling compression regions known as definite signatures of substorms were explained exactly (Ugai and Zheng 2006). In addition, we demonstrated that the magnetospheric current wedge drastically evolves when the fast reconnection jet collides with the magnetic loop (Ugai and Kondoh 2006).

Here, we are interested in flow enhancements in the near-Earth plasma sheet which are called bursty bulk

*Correspondence: kondo@cosmos.ehime-u.ac.jp

Research Center for Space and Cosmic Evolution, Ehime University,

Bunkyo-cho 3, Matsuyama 790-8577, Japan flows (BBFs) (Angelopoulos et al. 1992) and reverse flow (Chen and Wolf 1999; Panov et al. 2010). These earthward fast flows are associated with an increase in the northward magnetic field component, $B_{z}$, called dipolarization (Baumjohnn et al. 1990; Angelopoulos et al. 1992; Runov et al. 2009; Fujita and Tanaka 2013), and it includes a sharp increase in $B_{z}$ (a dipolarization front) (Runov et al. 2009). The evolution of the dipolarization front in the course of the earthward and rebound tailward flow is an unresolved problem.

The main theme of the present paper is to study the flow reversal using MHD simulations on the basis of the spontaneous fast reconnection model. Birn et al. (2011) illustrated properties of fast earthward flows using 3D MHD simulations. Recent multi-point observations have shown the importance of the observation position (Kondoh et al. 2011; Nakamura et al. 2005). In other words, observations made by satellites located at different positions vary significantly, even if the same event is observed simultaneously. In this paper, we investigate the time variations in physical quantities in different 3D positions by employing several virtual satellites located within our simulation domain.

\section{是 Springer}

(c) 2014 Kondoh and Shimizu; licensee Springer. This is an Open Access article distributed under the terms of the Creative Commons Attribution License (http://creativecommons.org/licenses/by/4.0), which permits unrestricted use, distribution, and reproduction in any medium, provided the original work is properly credited. 


\section{MHD simulations}

The simulation model in this paper is the same as that in our previous simulations of the three-dimensional magnetic loop (Kondoh et al. 2011) except for the initial thickness of the magnetic lobe region. In this study, we focus on the flow reversal associated with the bursty bulk flow in the near-Earth plasma sheet. Short-term magnetic reconnection is simulated with a thinner magnetic lobe region compared to that in our previous simulations.

\section{Simulation modeling}

Previous simulations of the magnetic loop using the wall boundary instead of the geomagnetic dipole field have shown that the fast reconnection jet in the plasma sheet is suddenly braked at that boundary because of the pressure force, and these results are in good agreement with the situation inferred from the observational results (Shiokawa et al. 1997). We focus on the evolution of plasma bulk flows, and the MHD approximations are valid for these macroscopic phenomena. The modified Lax-Wendroff scheme is used for numerical computation (Ugai 2008). Our coordinate system was chosen such that $x, y$, and $z$ directions are positive earthward, duskward, and northward, respectively.

As an initial configuration, a one-dimensional antiparallel magnetic field, $\mathbf{B}=\left[B_{x}(z), 0,0\right]$ was assumed, the same as for Kondoh et al. (2011). The initial ratio of the plasma pressure to the magnetic pressure in the lobe region was $\beta_{0}=0.15$; also, a constant temperature $T=1+\beta_{0}$ was assumed with a fluid velocity, $\mathbf{u}=(0,0,0)$. The normalization of quantities, based on the initial quantities, is self-evident: distances were normalized by the half thickness of the current sheet, $d_{0} ; \mathbf{B}$, by the field strength in the lobe region, $B_{x 0} ; P$, by $B_{x 0}^{2} /\left(2 \mu_{0}\right)$; $\rho$, by $\rho_{i}=\rho(z=0)$; $\mathbf{u}$, by $V_{A 0}\left(=B_{x 0} / \sqrt{\mu_{0} \rho_{i}}\right)$; time $t$, by $d_{0} / V_{A 0}$; current density $\mathbf{J}$, by $J_{0}=B_{x 0} /\left(\mu_{0} d_{0}\right)$. Note that the Alfvén velocity in the lobe region in the initial state is given by $V_{A e}=V_{A 0} / \sqrt{\rho_{e}}$ ( $\rho_{e}$ is the density in that region).

The axis symmetry conditions with respect to the $x=$ $0, z=0$ planes are assumed. With respect to the $x$ axis, for example, $\rho(x,-y,-z)=\rho(x, y, z), u_{x}(x,-y,-z)=u_{x}$ $(x, y, z), u_{y}(x,-y,-z)=u_{y}(x, y, z), u_{z}(x,-y,-z)=-u_{y}$ $(x, y, z), B_{x}(x,-y,-z)=-B_{x}(x, y, z), B_{y}(x,-y,-z)=B_{y}(x$, $y, z), B_{z}(x,-y,-z)=B_{z}(x, y, z)$, and $P(x,-y,-z)=P(x$, $y, z)$, for a fixed value of $x$ in $0 \geq x \geq L_{x}$. For simplicity, the same condition is assumed on the outer boundary plane $x=L_{x}$, whereas on the other boundary planes $\left(y=0, y=L_{y}\right.$, and $z=L_{z}$ ) the free boundary condition is assumed. Sufficiently small mesh sizes are required for precise computations of the spontaneous fast reconnection evolution, so we assumed $\Delta x=0.04, \Delta y=0.2$, and $\Delta z=0.015$. Furthermore, the magnetic lobe region size was assumed to be $Z_{1}=2$, and dimensions of the entire computation region were assumed to be $L_{x}=14, L_{y}=40$, and $L_{z}=9.6$. In our previous study, a larger $Z_{1}$ value was taken.

As in the previous studies, the anomalous resistivity model is assumed to be of the form

$$
\eta(\mathbf{r}, t)= \begin{cases}k_{R}\left[V_{d}(\mathbf{r}, t)-V_{C}\right] & \left(V_{d} \geq V_{C}\right) \\ 0 & \left(V_{d}<V_{C}\right)\end{cases}
$$

where $V_{d}(\mathbf{r}, t)=|\mathbf{J}(\mathbf{r}, t) / \rho(\mathbf{r}, t)|$ is the relative electron-ion drift velocity, and $V_{C}$ is the micro-instability threshold. In order to disturb the initial configuration, a localized resistivity is assumed at the origin in the $3 \mathrm{D}$ form

$$
\eta(\mathbf{r}, t)=\eta_{0} \exp \left[-\left(x / k_{x}\right)^{2}-\left(y / k_{y}\right)^{4}-\left(z / k_{z}\right)^{4}\right]
$$

Here, we take $k_{R}=0.003, V_{C}=12, k_{x}=k_{z}=0.8, k_{y}=5$, and $\eta_{0}=0.02$. The disturbance, Equation (2), is imposed only in the initial time range, $0 \leq t<4$, and the anomalous resistivity model, Equation (1), is assumed for $t \geq 4$.

\section{Results}

\section{General remarks}

Initiated by the disturbance, Equation (2), imposed around the origin in the finite extent, $|y|<k_{y}=5$, the current sheet thinning occurs, giving rise to a distinct enhancement of the current density. Once the ionelectron drift velocity, $V_{d}$, exceeds the threshold, $V_{C}$, at time $t \sim 20$, fast reconnection drastically grows as a nonlinear instability because of the positive feedback between the microscopic effective anomalous resistivity and the macroscopic reconnection flow. Figure 1 shows the temporal variation in the maximal $y$-component of the electric field in the diffusion region, $E_{y \max }=\left(\eta J_{y}\right)_{\max }$, where $E_{y}$ may indicate the effective reconnection rate. The magnetic reconnection grows drastically around $t=25$. Then, it suddenly decays at $t=30$ because of the bifurcation of the diffusion region in the $x$-direction and then recovers again around $t=34$. After that, magnetic reconnection gradually decays and terminates. On the other hand, around $t=42$, high pressure plasma in front of the dipolarization front collides with the wall boundary, across which plasma cannot flow, and the reconnected field lines are piled up, giving rise to a large-scale magnetic loop. The solid lines in Figure 2a,b show the magnetic field lines on the $x-z$ plane at $t=36$ and $t=42$, before and after the magnetic loop formation, respectively. The red and blue lines show the reconnected and non-reconnected magnetic field lines, respectively. In Figure $2 b$, the red lines inside of the thick magenta line are the magnetic loops.

\section{Flow reversal}

In order to investigate the time variation of the magnetic loop and plasma flow, Figure 2 shows the plasma flow 


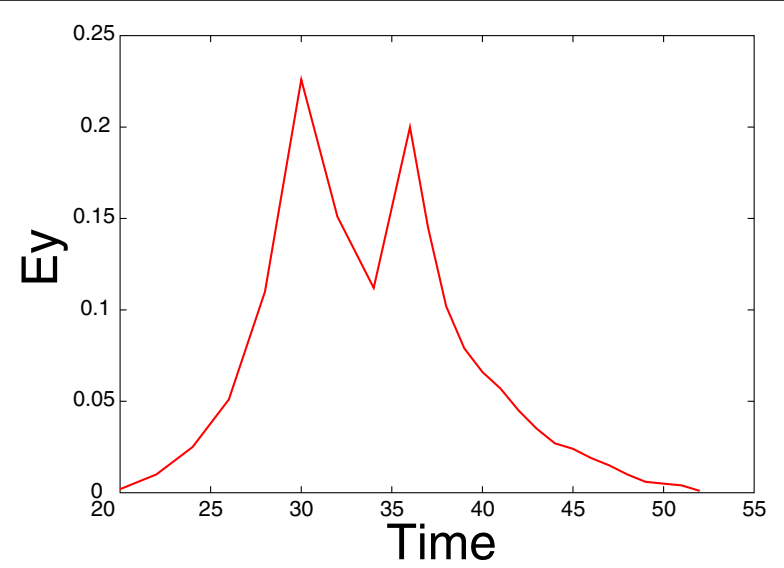

Figure 1 The time variation in the reconnection electric field $E_{y}$ at each time.

velocity, $V$ (arrows), and the magnetic field component, $B_{z}$ (black solid lines), on the $x-y$ plane at $t=36$ (a), $t=42$ (b), $t=48$ (c), and $t=54$ (d). The red (blue) arrows show positive (negative) $V_{x}$. The tailward flow region shown by the blue arrows expands with time from $t=42$. As it expands in the negative $x$-direction, the dipolarization front also moves tailward. Although the high $B_{z}$ region expands in the $y$-direction, the higher $B_{z}$ region behind the dipolarization front does not expand.
In order to show the rebounce of the dipolarization front, the second panel of Figure 3 shows the profiles of $B_{z}$ along the $x$-axis at times $t=36$ (red solid line), $t=42$ (green dashed line), $t=48$ (blue dotted line), and $t=54$ (magenta dotted line). On the $x$-axis, the dipolarization front moves earthward at $t=36$, then it clearly moves tailward at $t=54$.

\section{Virtual observations}

Our previous study showed that the time profiles of in situ observation data depend on the satellite position relative to the central line of the BBF (Kondoh et al. 2011). In order to investigate the similar dependence in the case of the reverse flow, we show in the Figures 4 and 5 the time profiles of the $x$-component of plasma flow velocity, $V_{x}$; the $z$-component of magnetic field, $B_{z}$; plasma density, $N$; and plasma pressure, $P$, at 16 different positions in the simulation domain $(x=10.0,10.8,11.6,12.4 ; y=0,2$; $z=0.0,0.75)$. These positions are shown in Figure 2a.

\section{On the neutral sheet ( $z=0.0$ plane)}

At first, we show the time profiles observed at four positions on the $x$-axis in Figure 4a. The $x$-position in our simulation coordinate system is equivalent to the distance from an $x$-line. The vertical solid and dashed lines in each panel indicate the time when the satellite observed the $B_{z}$ peak in the course of BBF and reverse flow, respectively.

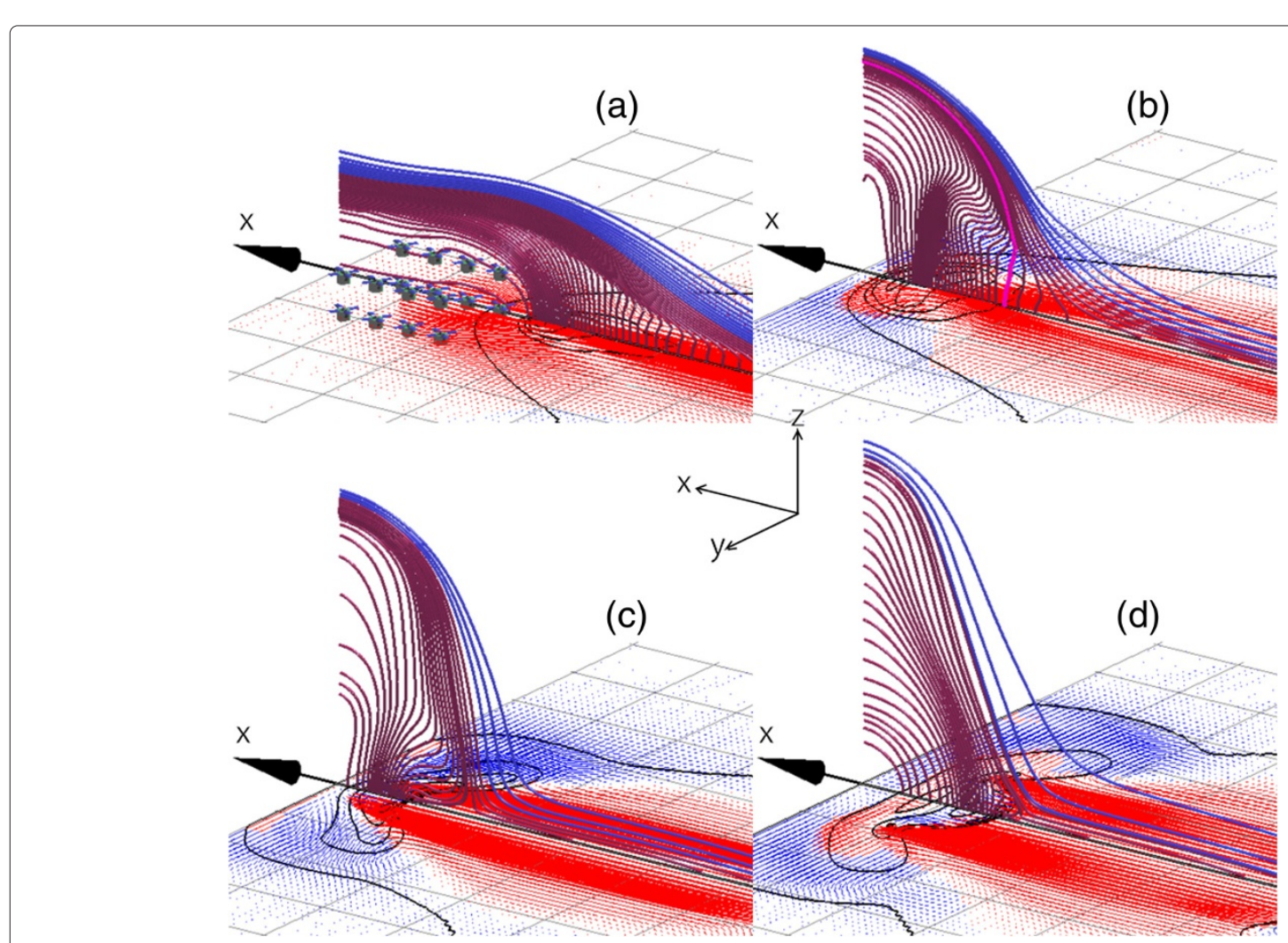

Figure 2 Magnetic field lines and plasma flows at four different times: $t=36(a), 42(b), 48(c)$, and 54(d). 


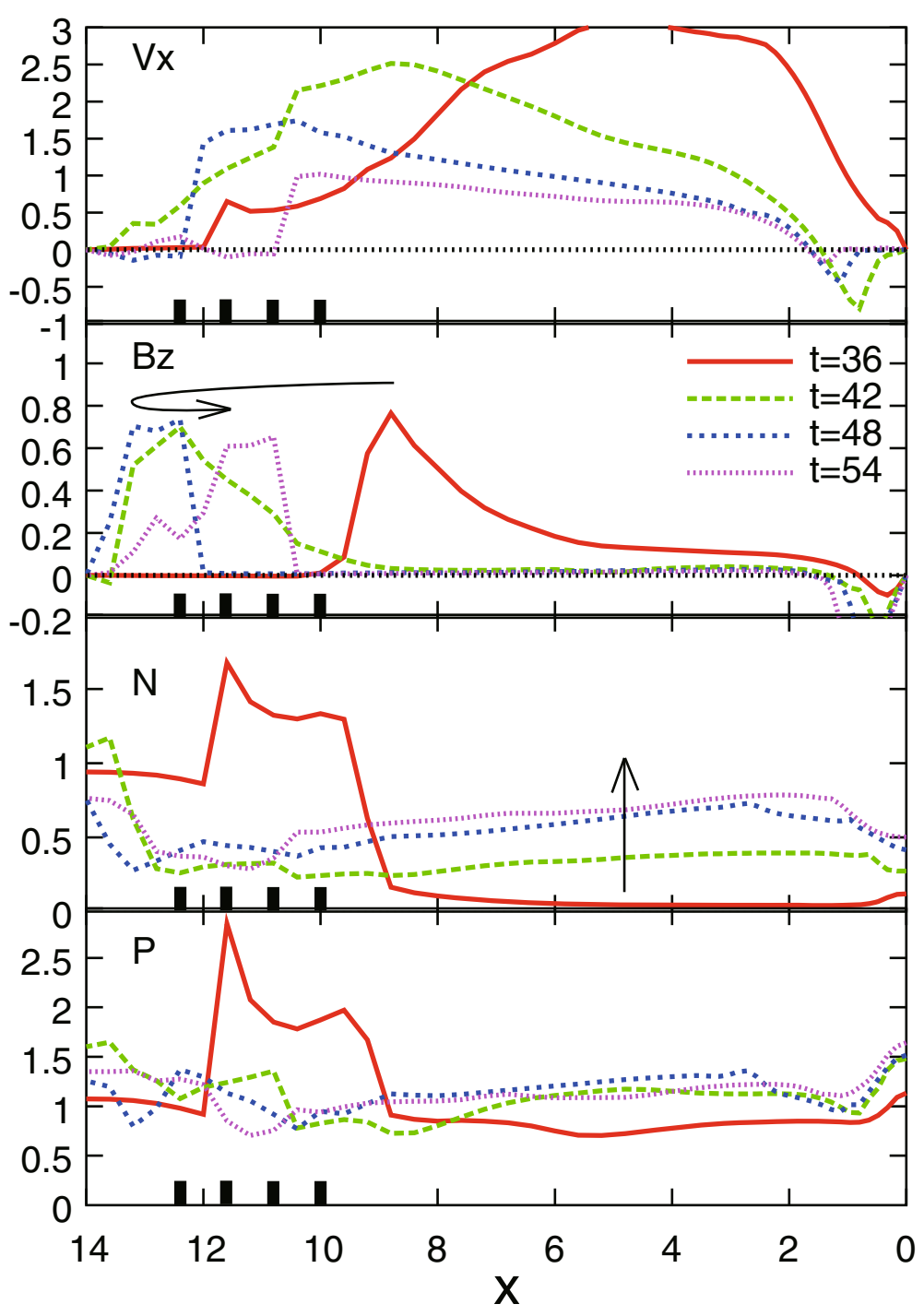

Figure 3 Profiles of quantities along the $x$-axis at time $t=36, t=42, t=48$, and $t=54$.

The reverse flow reaches $x=10.0$ at the end of the period in this panel, therefore there is no a blue dashed line. As shown in the previous paper (Kondoh et al. 2011), all satellites observe the rapid increase of $N$ and $P$ in the course of BBF just before that of $B_{z}$, while the peak values of $V_{x}$ in the course of BBF are detected later. On the other hand, in the course of reverse flow, only the increase of $B_{z}$ is significant, whereas that of $N$ and $P$ are insignificant. The sign reversal of $V_{x}$ is detected from the large $x$-position (the nearest position to the wall boundary). The small enhancements in the $N$ and $P$ profiles are seen between the vertical solid and dashed lines.

Next, time profiles observed at the positions off the $x$ axis in the $y$-direction, $y=2$, are shown in Figure $4 b$, using the same format as Figure 4a. The $y$-position in our simulation coordinate system is equivalent to the distance from the central line of the fast flow channel in the $y$ direction. As shown in the previous paper (Kondoh et al. 2011), the variations in all values are gradual and small, and the peak values of $V_{x}$ in the course of BBF are detected later than those in Figure 4a, except for the observation at $x=12.4$, which does not detect any significant enhancement in $V_{x}$. In spite of this lack of significant enhancement in $V_{x}$ at $x=12.4$, the enhancement in $B_{z}$ is large and long. In addition, the interval between these two $B_{z}$ peaks becomes longer in Figure $4 \mathrm{~b}$.

\section{Off the neutral sheet $(z=0.75$ plane)}

The $z$-position in our simulation coordinate system is equivalent to the distance from the neutral sheet. Figure 5 


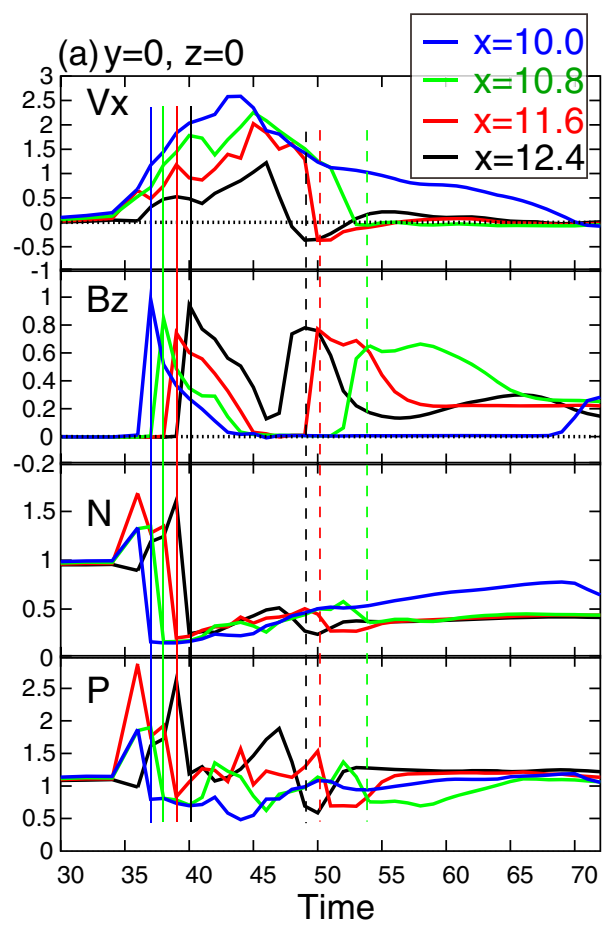

(b) $y=2, z=0$

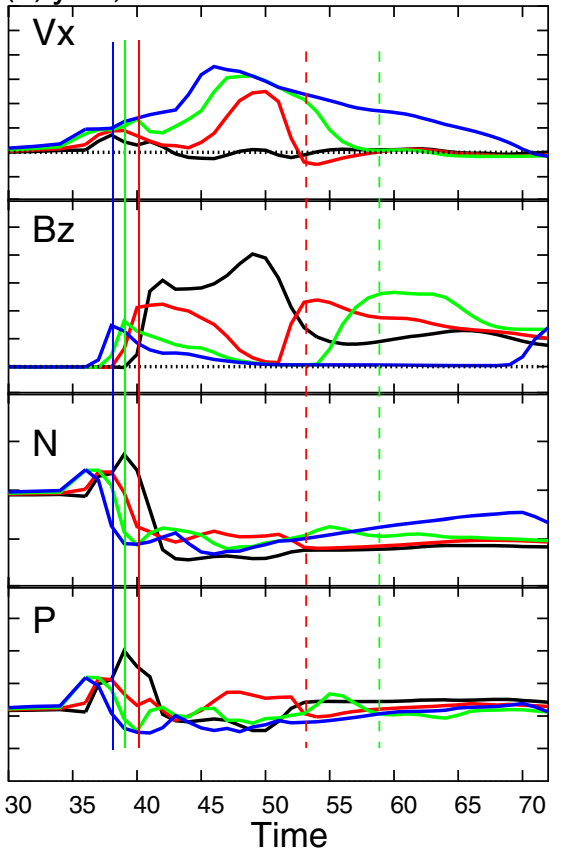

Figure 4 Time profiles of $V_{x}, B_{z}, N$, and $P$ at $x=10.0$ (blue lines), $x=10.8, x=11.6$, and $x=12.4 ; y=0$ (a) and $y=2$ (b); and $z=0$.

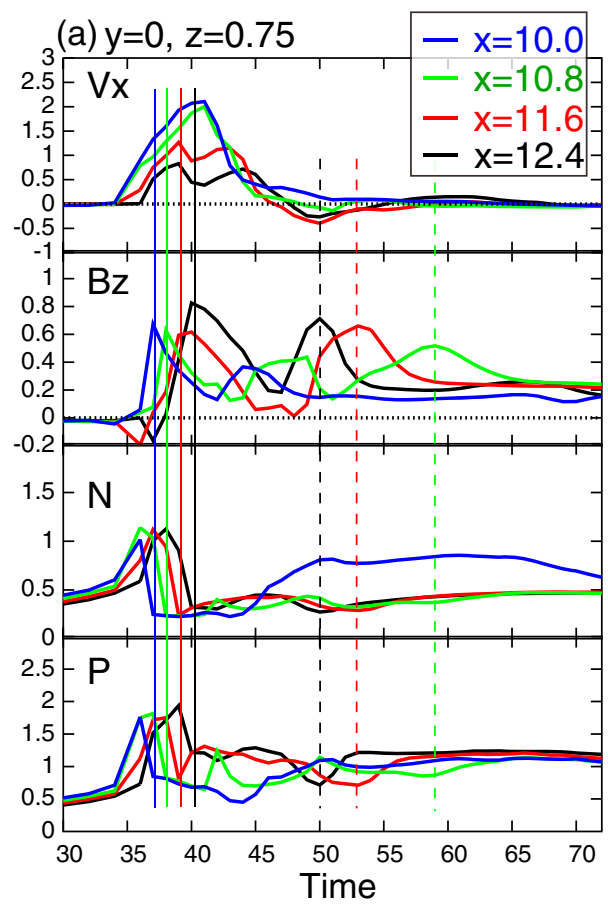

(b) $y=2, z=0.75$

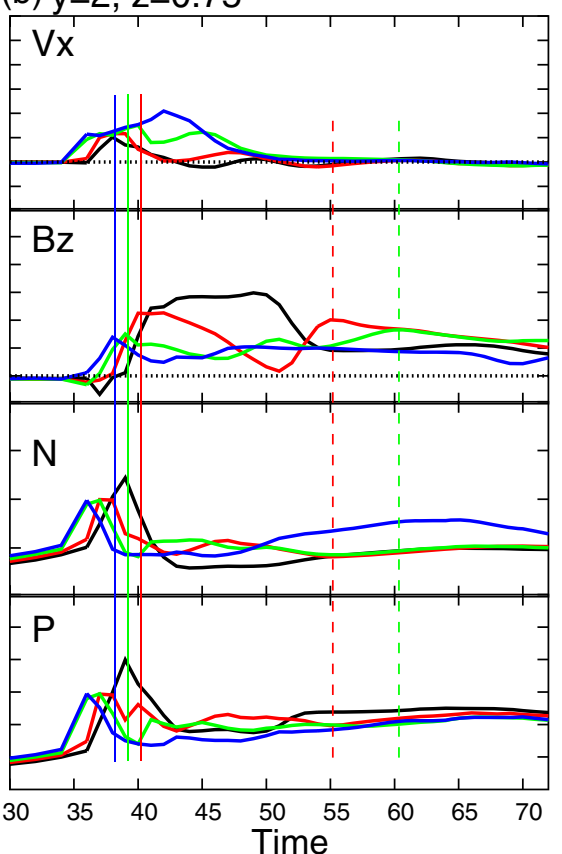

Figure 5 Time profiles of the same quantities as for Figure $\mathbf{4}$ observed on the $\boldsymbol{z}=\mathbf{0 . 7 5}$ plane. (a) $y=0$, (b) $y=2$. 
shows variations in the same parameters as those shown in Figure 4, observed at $z=0.75$. Small and short enhancements in $V_{x}$ and small enhancements in $P$, compared to those observed around the neutral sheet, are seen in Figure 5. The interval between the peak detections of $V_{x}$ and that of $B_{z}$ becomes longer than that on the neutral sheet. The detection of the sign reversal of the $x$ component for the velocity, $V_{x}=0$, is random. The small enhancements between the vertical solid and dashed lines in the $N$ and $P$ profiles are also seen at positions off the neutral sheet.

\section{Summary and discussion}

In this paper, the spontaneous fast reconnection model was applied to the earthward fast flow and the reverse flow problem observed in the near-Earth plasma sheet. Once the current-driven anomalous resistivity is ignited, the positive feedback between the anomalous resistivity and the reconnection flow gives rise to the 3D fast reconnection mechanism. The dipolarization front is formed from the accumulated reconnected magnetic field lines in front of the fast reconnection jet. As a result of the collision with the wall boundary, a large magnetic loop is formed, and the magnetic loop (the dipolarization front) rebounces tailward.

In this study, time variations in these earthward and tailward fast flows were investigated by employing virtual satellites located at different positions in the MHD simulation space. On the center of the earthward fast flow (on the $x$-axis in our simulations), the peak of the velocity component, $V_{x}$, enhancement in the course of the BBF is detected later than that of the magnetic field component, $B_{z}$, plasma density, $N$, and plasma pressure, $P$, enhancement. These time lags are much longer at positions off the center line of the fast flow channel because the reconnection rate (reconnection electric field) on the $x$-line is largest at the center in the $y$-direction (see Ugai and Kondoh (2003) for details).

On the other hand, these time lags become shorter at positions off the neutral sheet in the $z$-direction because of the displacement of the satellite from the fast flow channel due to the plasma sheet thinning. As a result of these dependencies on the satellite position, the interval between the peak of $V_{x}$ enhancement in the course of the BBF and that of the $V_{x}$ decrease in the course of the reverse flow does not have a good correlation with the radial distance of the satellite from the center of the Earth.

The interval between the peaks of $B_{z}$ enhancements in the course of the BBF and reverse flow appear to have a good correlation with the radial distance of the satellite, but the peak of the enhancement delays at a position far from the center of the flow channel in the $y$-direction. Therefore, the good correlation between the interval and the radial distance of the satellite cannot be obtained without the consideration of this delay.

The small enhancements in $N$ and $P$ observed by a satellite are observed by nearly all of the virtual satellites in the interval between the above two peaks of $B_{z}$ enhancements. The third and bottom panels of Figure 3 show the profiles of $N$ and $P$ along the $x$-axis. The small black symbols on the $x$-axis in these panels indicate the position of virtual satellite observations. $N$ and $P$ gradually recover after the passage of the dipolarization front, as shown by the arrow in the third panel. These decrease again during the passage of the dipolarization front. Therefore, $N$ and $P$ increase in the interval between the $B_{z}$ peaks. In future work, we will verify these simulation results using observational data from in situ satellites.

\section{Competing interests}

The authors declare that they have no competing interests.

\section{Authors' contributions}

KK and TS carried out the space plasma studies. KK drafted the manuscript. Both authors read and approved the final manuscript.

\section{Acknowledgements}

This work was supported by the Research Institute of Sustainable Humanosphere (RISH), Kyoto University, and the Solar Terrestrial Environment Laboratory (STELab), Nagoya University. The computer program was tested and run at the computational centers of Nagoya University and Kyoto University.

Received: 31 March 2014 Accepted: 15 August 2014

Published online: 29 October 2014

References

Angelopoulos V, Baumjohnn W, Kennel CF, Voroniti FV, Kivelson MG, Pellat R, Walker RJ, Luhr H, Paschmann G (1992) Bursty bulk flows in the inner central plasma sheet. J Geophys Res 97:4027-4039

Baumjohnn W, Paschmann G, Luhr H (1990) Characteristics of high-speed ion flows in the plasma sheet. J Geophys Res 95:3801-3809

Birn J, Panov EV, Hesse M (2011) Bursty bulk flows and dipolarization in MHD simulations of magnetotail reconnection. J Geophys Res 116:A01210

Chen CX, Wolf RA (1999) Theory of thin-filament motion in Earth's magnetotail and its application to bursty bulk flows. J Geophys Res 104:14,613-14,626. doi:10.1029/1999JA02080

Fujita S, Tanaka T (2013) Possible generation mechanisms of the Pi2 pulsations estimated from a global MHD simulation. Earth Planets Space 65:453-461

Kondoh K, Ugai M, Shimizu T (2011) Multi-point observations of earthward fast flow in the plasma sheet by virtual satellites located in the MHD simulation domain. Adv Space Res 48:287-291

Nakamura R, Amm O, Laakso H, Draer NC, Lester M, Grocott A, Klecker B, McCrea IW, Balogh A, Reme H, Andre M (2005) Localized fast flow disturbance observed in the plasma sheet and in the ionosphere. Ann Geophys 23:553-566

Panov EV, Nakamura R, Baumjohann W, Sergeev VA, Petrukovich AA, Angelopoulos $V$, Volwerk M, Retino A, Takada T, Glassmeier KH, McFadden JP, Larson D (2010) Plasma sheet thickness during a bursty bulk flow reversal. J Geophys Res 115:A05213

Runov A, Angelopoulos V, Sitnov MI, Sergeev VA, Bonnell J, McFadden JP, Larson F, Glassmeier K, Auste U (2009) THEMIS observations of an earthward propagating dipolarization front. J Geophys Res Lett 36:L14106

Shiokawa K, Baumjohann W, Haerendel G (1997) Braking of high-speed flows in the near-Earth tail. Geophys Res Lett 24:1179-1182

Ugai M, Kondoh K (2006) Evolution of magnetospheric current wedge by the spontaneous fast reconnection model. Phys Plasmas 13:102903 
Ugai $M$, Zheng $L$ (2006) Modeling of traveling compression regions in the Earth's magnetotail by the spontaneous fast reconnection model. Phys Plasmas 13:102901

Ugai M (2008) Evolution of fast reconnection in a three-dimensional current sheet. Phys Plasmas 15:082306

Ugai M, Kondoh K (2003) Computer simulations on three-dimensional magnetic loop dynamics by the spontaneous fast reconnection model. Phys Plasmas 10:357

doi:10.1186/s40623-014-0147-1

Cite this article as: Kondoh and Shimizu: Study of plasma flow reversal in the near-Earth plasma sheet using numerical computations. Earth, Planets and Space 2014 66:147.

Submit your manuscript to a SpringerOpen ${ }^{\circ}$ journal and benefit from:

- Convenient online submission

- Rigorous peer review

- Immediate publication on acceptance

- Open access: articles freely available online

- High visibility within the field

- Retaining the copyright to your article

Submit your next manuscript at springeropen.com 